\title{
Article \\ Combined Transcriptome and Metabolome Analysis of Alfalfa Response to Thrips Infection
}

\author{
Zhiqiang Zhang ${ }^{1,2}$, Qi Chen ${ }^{1}$, Yao Tan ${ }^{3}$, Shuang Shuang ${ }^{1}$, Rui Dai ${ }^{2}$, Xiaohong Jiang ${ }^{1}$ and Buhe Temuer ${ }^{1,2, *}$ \\ 1 Key Laboratory of Grassland Resources of the Ministry of Education, Technology Engineering Center of \\ Drought and Cold-Resistant Grass Breeding in North of the National Forestry and Grassland Administration, \\ College of Grassland, Resources and Environment, Inner Mongolia Agricultural University, \\ Hohhot 010011, China; zhangzq1989@imau.edu.cn (Z.Z.); ndcq@emails.imau.edu.cn (Q.C.); \\ bss@emails.imau.edu.cn (S.S.); jxh19971002@sina.com (X.J.) \\ 2 Key Laboratory of Grassland Resources of the Ministry of Education, College of Grassland, Resources and \\ Environment, Inner Mongolia Agricultural University, Hohhot 010011, China; 18747659997@163.com \\ 3 College of Horticulture and Plant Protection, Inner Mongolia Agricultural University, Hohhot 010011, China; \\ 850310.tanhuaf4@163.com \\ * Correspondence: te1988@imau.edu.cn; Tel.: +86-0471-4316259
}

check for

updates

Citation: Zhang, Z.; Chen, Q.; Tan, Y.; Shuang, S.; Dai, R.; Jiang, X.; Temuer, B. Combined Transcriptome and Metabolome Analysis of Alfalfa Response to Thrips Infection. Genes 2021, 12, 1967. https://doi.org/ $10.3390 /$ genes 12121967

Academic Editors: Zhipeng Liu, Wenxian Liu, Zhen Wang and Valérie Geffroy

Received: 4 November 2021 Accepted: 7 December 2021 Published: 10 December 2021

Publisher's Note: MDPI stays neutral with regard to jurisdictional claims in published maps and institutional affiliations.

Copyright: (c) 2021 by the authors. Licensee MDPI, Basel, Switzerland. This article is an open access article distributed under the terms and conditions of the Creative Commons Attribution (CC BY) license (https:/ / creativecommons.org/licenses/by/ $4.0 /)$.

\begin{abstract}
Thrips (Thysanoptera: Thripidae) is a major insect pest for alfalfa which can result in decreased plant nutrients, low yields, and even plant death. To identify the differentially expressed genes and metabolites in response to thrips in alfalfa, a combination of metabolomics and transcriptomics was employed using alfalfa (Caoyuan No. 2) with and without thrips infestation. The results showed that the flavonoid biosynthesis and isoflavonoid biosynthesis pathways were the most significantly enriched pathways in response to thrips infection, as shown by the combined transcriptome and metabolome analysis. The transcriptome results showed that SA and JA signal transduction and PAPM-triggered immunity and the MAPK signaling pathway-plant pathways played a crucial role in thrips-induced plant resistance in alfalfa. In addition, we found that thrips infestation could also induce numerous changes in plant primary metabolism, such as carbohydrate and amino acid metabolism as compared to the control. Overall, our results described here should improve fundamental knowledge of molecular responses to herbivore-inducible plant defenses and contribute to the design of strategies against thrips in alfalfa.
\end{abstract}

Keywords: transcriptome; metabolome; Medicago sativa; thripidae; induced defense

\section{Introduction}

Alfalfa (Medicago sativa L.), a legume forage with high quality and yield, has become an important material basis for the development of grass husbandry all over the world. Herbivorous thrips (Thysanoptera) are important pests affecting alfalfa in China and most other regions where alfalfa is grown [1,2]. Thrips feed on leaves, stems, fruits, and/or pollen of green plants, and they are vectors of destructive viruses, resulting in decreased plant nutrients and growth, low yields, and even plant death [3]. It has been reported that thrips cause about $10 \%-30 \%$ grass yield loss every year [4]. However, thrips are highly polyphagous and hard to control due to their complex lifestyle [3]. Integrated pest management guidelines for crops emphasize use of a range of tactics to reduce pest abundance, rather than reliance on insecticides, which would induce ecosystem damage and food safety issues [5].

Plants have developed highly effective and dynamic defensive strategies against insect pests, including various morphological and biochemical defenses that restrict insect pests, including constitutive defense and induced defense [6,7]. Compared with constitutive defense, induced defense is more often initiated upon herbivore attack due to the trade-off between plant growth and defense [8]. Herbivore-inducible plant defenses are initiated after perception of the herbivore through damage-associated molecular patterns or 
herbivore-associated molecular patterns. Many authors have reported that several morphological traits were associated with host plant resistance to thrips, such as leaf hair density, leaf hairiness, leaf hardness, leaf wax, glandular hairs, and trichomes [9-11]. However, biochemical-based defense is considered more effective, as it directly affects insect growth and development [12]. It has been reported that many metabolites and plant hormones are associated with thrips resistance, such as protease inhibitors, phenols, tannins, salicylic acid (SA), and jasmonic acid (JA) [13-16]. Most of the metabolites known to be involved in thrips constitutive defense are inherently present in the plant [17-21], but some accumulate in response to thrips infestation. For instance, phenolic compounds, such as tannin, have been found to accumulate in response to thrips infection $[7,20]$.

Compared to leaf-chewing or phloem-feeding herbivores, far less is known about the induced plant responses to cell-content-feeding insects such as thrips [8]. Even though there is literature related to constitutive defense mechanisms against thrips [17-19,21], functional analyses of defensive compounds that are induced upon thrips attack are less $[3,20]$. Next generation sequencing, and more specifically RNA-Sequencing (RNA-Seq), has become a popular and comprehensively informative approach to predict and validate novel key regulators and their direct and indirect targets in plant signaling networks to pests. Recently, a comparative transcriptomes analysis was employed to assemble the expressed genes of alfalfa, which mainly focused on the induced defense genes related to both resistant and susceptible alfalfa lines after thrips infestation, but the analysis paid little attention to the genes specially related to thrips resistance in the resistant cultivar [22]. In addition, metabolomics can aid the discovery of plant metabolites related to thrips induced resistance. Combined transcriptome and metabolome can allow quantitative mapping of transcripts directly to metabolic pathways involved in thrips induced resistance.

It is important to understand the metabolic changes, transcriptional regulation, and physiological responses of bioactive and signaling compounds during infection of alfalfa with thrips. Thus, to identify the differentially expressed genes and metabolites in response to thrips in alfalfa, a combination of metabolomics and transcriptomics was employed using alfalfa (Caoyuan No. 2) with and without thrips infestation. The results showed that the flavonoid biosynthesis and isoflavonoid biosynthesis pathways were the most significantly enriched pathways in response to thrips infection, as shown by the combined transcriptome and metabolome analysis. The transcriptome results showed that plant hormones signal transduction (SA and JA) PAPM-triggered immunity and that MAPK signaling pathway-plant pathways played a crucial role in thrips-induced plant resistance in alfalfa. In addition, we found that thrips infestation could also induce numerous changes in plant primary metabolism, such as carbohydrate and amino acid metabolism as compared to the control. Overall, our results described here should improve fundamental knowledge of molecular responses to herbivore-inducible plant defenses and contribute to the design of strategies against thrips in alfalfa.

\section{Materials and Methods}

\subsection{Plant Growth and Thrips Infection}

Seeds of alfalfa (Caoyuan No. 2) were cultivated in pots $(\mathrm{H} 21 \mathrm{~cm} \times \mathrm{D} 14 \mathrm{~cm}$, one plant per pot) containing field collected soil in a greenhouse with a relative humidity of $60 \pm 5 \%$ and $70 \pm 5 \%$ at $30 \pm 5{ }^{\circ} \mathrm{C}$ and $20 \pm 5^{\circ} \mathrm{C}$ during day and night, respectively. Plants were watered every other day. Both cultivars were bred at Inner Mongolia Agricultural University, China. Alfalfa plants were treated as described by Tu et al. [22] with some modifications. When the seedlings reached budding stage (about 60 days), they were randomly and equally divided into two groups: (1) 30 alfalfa thrips per plant were placed onto the leaves and covered by a cage with a 90-mesh nylon cloth as the S_T treatment group; and (2) plants were not treated with thrips and were maintained under the same conditions, as the S_CK treatment group. Three plants were grown in each pot, and 4 pots were counted as one biological replicate. After three weeks, the top 3-4 leaves were cleaned 
of any thrips and harvested from each treatment. All samples were immediately frozen in liquid nitrogen and stored at $-80^{\circ} \mathrm{C}$.

\subsection{RNA Extraction, cDNA Library Construction and RNA-Sequencing}

Three biological replicates were used for all RNA-Seq experiments from thrips and no-thrips treatments. RNA extraction, cDNA library construction and RNA-sequencing were carried out as described by $[13,23]$. Briefly, total RNA was extracted from the leaves using Trizol reagent (Invitrogen, Carlsbad, CA, USA) along with DNase treatment, according to the manufacturer's instructions (QIAGEN, Hilden, Germany). The quality and quantity of total RNA were assessed using NanoDrop 2000 analysis and gel electrophoresis. As described by [24], the cDNA libraries were prepared using a TruseqTM RNA sample prep Kit (Illumina, San Diego, CA, USA), and RNA-sequencing was performed on an Illumina Hiseq 4000 (Version $2 \times 150 \mathrm{bp}$ ) at Shanghai Majorbio Bio-pharm Biotechnology Co., Ltd. (Shanghai, China). The raw sequence reads were deposited in the NCBI Sequence Read Archive (http://www.ncbi.nlm.nih.gov/Traces/sra, accession number PRJNA622603) (accessed on 3 April 2020).

\subsection{De Novo Assembly, Annotation and Classification}

The clean data were obtained by removing the adapter and primer sequences using SeqPrep software, and fragments of less than $20 \mathrm{bp}$ in length were excluded from further analyses using software Trinity (https://github.com/trinityrnaseq/trinityrnaseq) (accessed on 25 September 2021) in the absence of a reference genome [25]. The sequence assembly quality was evaluated using the number of sequences and bases, GC percentage, distribution of unigene lengths, average coverage, and N50 statistics [26].

The assembled transcriptome sequences were searched against six databases (NR, Swiss-Prot, Pfam, COG, GO, and KEGG databases) to obtain annotation information in each database. Specifically, to obtain the similarity to other species and the functional information of homologous sequences, the sequences were searched against the NCBI nonredundant database (NR, ftp:/ / ftp.ncbi.nlm.nih.gov/blast/db/, accessed on 25 September 2021), Swiss-Prot database (http:/ / web.expasy.org/docs/swiss-prot_guideline.html, accessed on 25 September 2021), and Pfam (http://pfam.xfam.org/, accessed on 25 September 2021). The gene function terms were obtained through the Gene Ontology database (GO, http:/ / www.geneontology.org, accessed on 25 September 2021). Functional classification was performed using the Clusters of Orthologous Groups of proteins database (COG, http:/ /www.ncbi.nlm.nih.gov/COG/, accessed on 25 September 2021), and pathway annotation was performed using the Kyoto Encyclopedia of Genes and Genomes (KEGG, http://www.genome.jp/kegg/, accessed on 25 September 2021). A $p$-value $\leq 0.05$ was regarded as the threshold for significance [26]. In addition, quantitative analysis of gene and transcript expression levels was obtained through RSEM (http:/ / deweylab.github.io/RSEM/, accessed on 25 September 2021), and principal component analysis (PCA) was performed to obtain the relationships among and variability between samples.

\subsection{Differentially Expressed Genes Analysis and Enrichment Analysis}

Differential expression gene analysis of the samples was performed using DEseq 2 software. A $p$-adjusted value $<0.05$ and $|\log 2 \mathrm{FC}| \geq 1$ was set as the threshold. To identify shared and unique genes/transcripts across gene sets, Venn analysis was performed. A gene set enrichment analysis of the differentially expressed genes (DEGs) was then performed for the KEGG annotations to determine over-represented functional pathways (with a $p$-value $<0.05$ ) at each comparison level for different genotypes and treatments. 


\subsection{Metabolome Analysis}

The sample processing, extraction, and metabolites detection for metabolome analysis were performed on Wuhan MetWare Biotechnology Co., Ltd. (Wuhan, China) following their standard procedures [27].

\subsection{Metabolomics Data Analysis}

Data matrices with the intensity of metabolite features with and without thrips infection were submitted and processed in Analyst 1.6.3 software (AB SCIEX, Concord, ON, Canada). The missing values were considered to be below the detection limit and imputed with a minimum recorded value [28]. The ion intensities were normalized by log transformation, metabolite abundance was calculated by using Dunnett's test, and multiple testing was controlled by fold change $\geq 2$ and fold change $\leq 0.5$. We used the quadrature signal correction partial least squares-discriminant analysis (OPLS-DA) and the variable importance in projection (VIP) to obtain the maximum differences between control and thrips infection. Metabolites with VIP $>1.0$ were considered as differential metabolites for group discrimination. The KEGG database [28] was used to annotate and display the differential metabolites. Other analyses included Principal Component Analysis (PCA) and pathway enrichment, which were completed using $\mathrm{R}$ as reported (www.rproject.org, accessed on 20 June 2020) [27].

\subsection{Combined Transcriptome and Metabolome Analyses}

We performed co-joint analyses on the differentially expressed genes and differentially accumulated metabolites to determine the degree of enrichment of pathways. Genemetabolite networks with a Pearson correlation coefficient (PCC) $>0.8$ were used to construct the transcript-metabolite network [29].

\section{Results}

\subsection{Summary of Transcriptome and Metabolome Analysis}

Transcription and widely targeted metabolites profiles of Caoyuan No. 2 without or with thrips treatment (S_CK vs. S_T) were explored. Three independent biological replicates were used for each treatment, resulting in six samples. A total of $86.34 \mathrm{~Gb}$ Clean Data was obtained and the clean data of each sample reached more than $12.57 \mathrm{~Gb}$, and the Q30 base percentage was more than $91.67 \%$. A transcriptome database containing 99,111 unigene of average length 822.74 bp was obtained using Trinity software, with an N50 length of $1267 \mathrm{bp}$ and an E90N50 length of $2172 \mathrm{bp}$. All unigenes and transcripts obtained by transcriptome assembly were aligned with six major databases $(\mathrm{Nr}$, Swiss-prot, Pfam, COG, GO, and KEGG databases). A total 62,266 homologs of the 99,111 assembled unigenes were found to have homologs in the databases NR $(56,433)$, Swiss-prot $(40,046)$, Pfam $(40,012)$, COG $(45,178)$, GO $(48,280)$, and KEGG $(26,953)$ (Figure 1A). For the species distribution of the top BLAST hits in the NR database, 39,403 (69.03\%) annotated unigenes matched the sequence of Medicago truncatula (Figure 1B). In addition, 772 metabolites were detected, which could be grouped into 23 major classes (Table S1).

Principal component analysis (PCA) of the differentially expressed genes and differentially accumulated metabolites showed that the S_CK treatment group showed obvious differences with the S_T treatment group, which explained $44.32 \%$ and $49.88 \%$ of the total variation (Figure 2A,B). These results indicated that Caoyuan No. 2 is susceptible to thrips. 

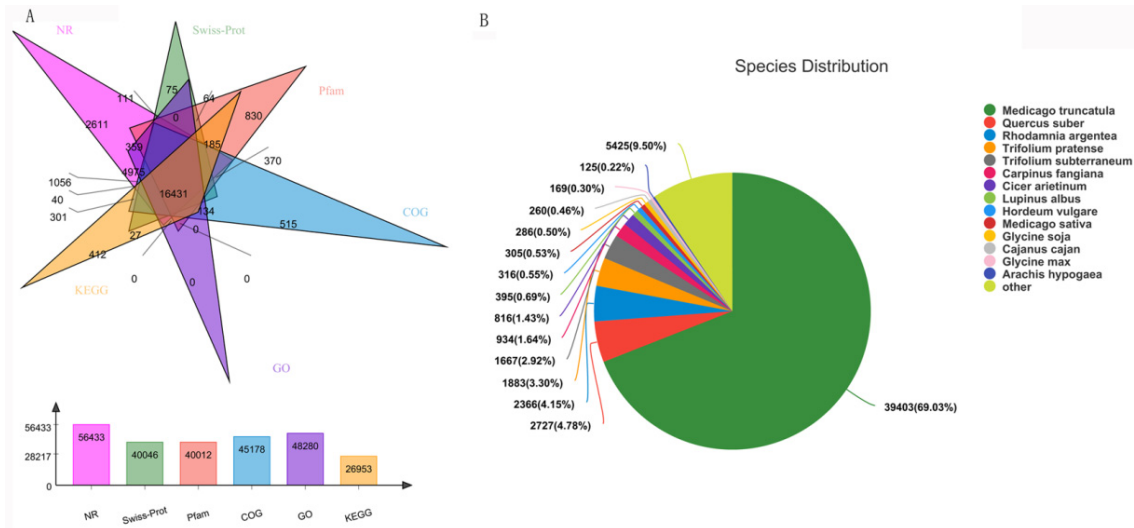

Figure 1. Function annotations of transcriptome sequencing. (A) Functional annotation numbers of unigenes in the NR, Swiss-prot, Pfam, COG, GO, and KEGG databases. (B) Annotated species distribution in the NR database.

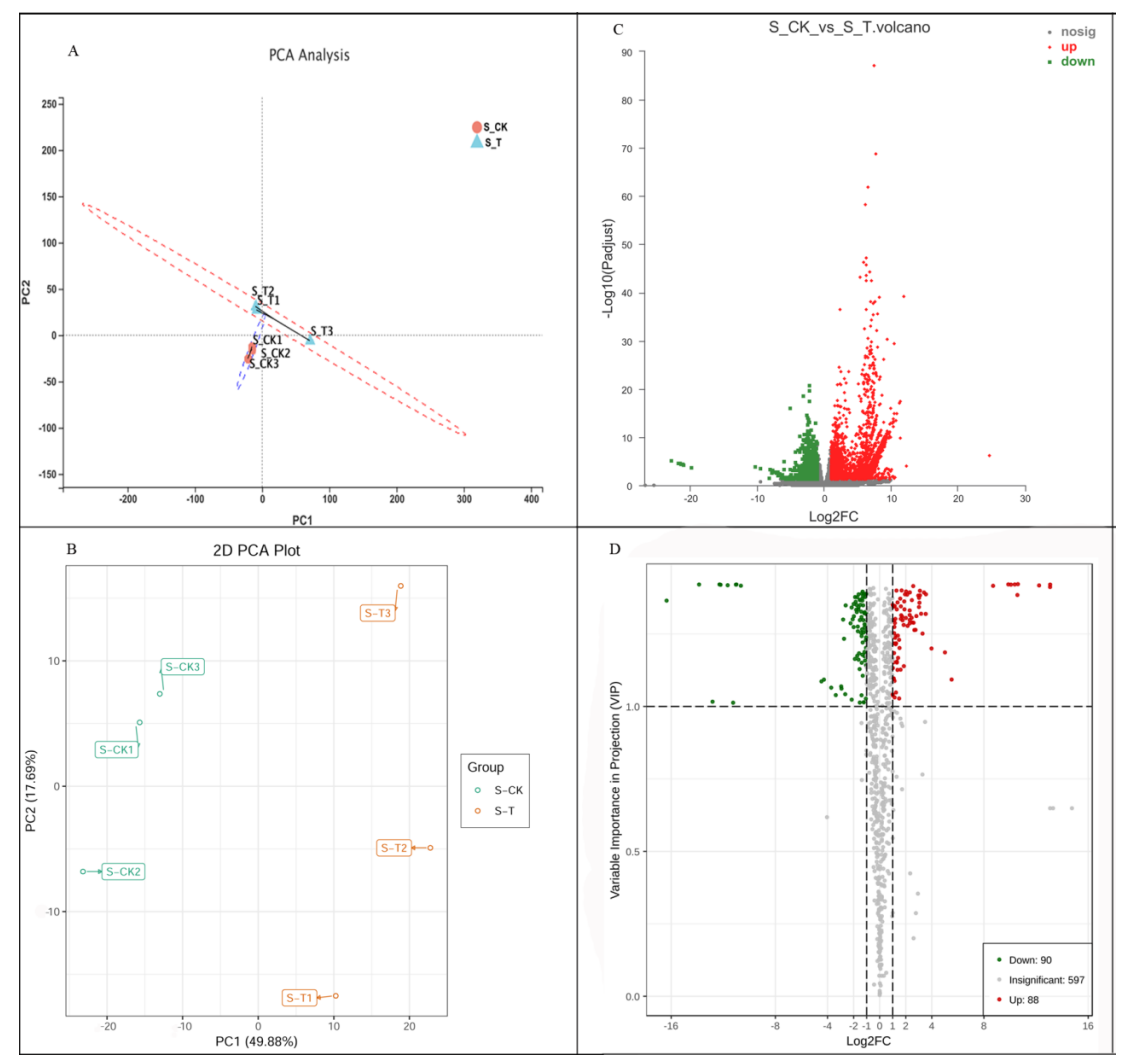

Figure 2. Differentially expressed genes and differentially accumulated metabolites between S_CK and S_T. (A) Principal component analysis (PCA) of the variance-stabilized estimated raw counts of differentially expressed genes. (B) Principal component analysis (PCA) of the variance-stabilized estimated raw counts of differentially accumulated metabolites. (C) Expression profiling changes of genes in thrips injection tissues. (D) Volcano Plot of differentially expressed genes between S_CK and S_T. Each point in the volcano map represents a metabolite, the $\mathrm{X}$ axis represents the logarithm of the quantitative difference of a certain metabolite in the two samples; the $\mathrm{Y}$ axis represents the VIP value. Samples are categorized by cultivars and thrips infestation as different marker colors and shapes. The green dots represent downregulated differentially expressed genes or differentially accumulated metabolites, the red dots represent upregulated differentially accumulated metabolites or genes, and the gray represents detected but not significantly differentially expressed genes or differentially accumulated metabolites. S_CK: Caoyuan No. 2 without thrips infection; S_T: Caoyuan No. 2 with thrips infection. 


\subsection{Differentially Expressed Genes and Differentially Accumulated Metabolites Analysis Related to Thrips Infection}

The differentially expressed genes among the two groups were analyzed in the RNAseq datasets. After treatment with thrips, a total of 4187 DEGs were detected, of which, respectively, 3379 and 808 upregulated and downregulated differentially expressed genes were observed (S_CK vs. S_T, $p$-adjust value $<0.05$ and $|\log 2 \mathrm{FC}| \geq 1$, Figure $2 \mathrm{C}$ ). For the evaluation of differentially accumulated metabolites between S_CK and S_T, the OPLS-DA model was applied. The established OPLS-DA model showed good fitness (Figure S1). After treatment with thrips, a total of 88 upregulated and 90 downregulated metabolites were detected between the treatments (S_CK vs. S_T, VIP $>1$ and $|\log 2 \mathrm{FC}| \geq 1$, Figure 2D).

Further analysis showed that 1681 upregulated and 199 downregulated genes were annotated to 116 and 74 different KEGG pathways, respectively (Table S2). Further analysis showed that 499 of the upregulated and 19 of the downregulated differentially expressed genes were annotated to pathways including genetic information process (such as folding, sorting, and degradation transcription, translation), 723 of the upregulated and 111 of the downregulated differentially expressed genes were related to metabolism (such as carbohydrate metabolism, lipid metabolism, amino acid metabolism, biosynthesis of other secondary metabolites, etc.), 80 of the upregulated and 10 of the downregulated differentially expressed genes were involved in cellular process, and 41 and 29 upregulated as well as 9 and 10 of the downregulated differentially expressed genes were annotated to environmental adaptation and signal transduction, respectively (Figure 3A,B, Table S2).

Furthermore, we found that all the key genes (NPR1, TGA, and PR-1) related to salicylic acid (SA) transduction and JAZ gene related to jasmonic acid (JA) signal were significantly induced in S_CK after thrips attack (Figure S2). In addition, the results showed that some key genes involved in plant-pathogen interaction (such as elfl8, CDPK, Rboh, CaMCML, MPK4, WRKY22, WRKY25, WRKY29, WRKY33, Pit6, HSP90, SGT1, EDS1, NHO1, $P R 1$, and KCS) and MAPK signaling pathway-plant (such as MPK4, WRKY22, WRKY29, WRKY33, ACS6, NDPK2, CaM4, Rboh, and PR1) were induced or suppressed by thrips infection (Figures S3 and S4).

For differentially accumulated metabolites, we found that the differentially accumulated metabolites were most significantly enriched to flavonoid biosynthesis, isoflavonoid biosynthesis, amino acids biosynthesis, and arginine and proline biosynthesis (Figure 4A). Furthermore, the results showed that the top 10 upregulated metabolites in alfalfa after thrips infection were 8-Hydroxy-2-deoxyguanosine (pmb3350), 4-O-Caffeoyl quinic acid (pme2938), genistein (pme1578), 3,7-Di-O-methylquercetin (pme3288), 8-Hydroxy2-deoxyguanosine (pme3350), pinocembrin (pme2982), tricetin (pme3303), L-Carnosine (pme0116), tricin O-phenylformic acid (pmb0744), 1-O-p-Coumaroyl quinic acid (pmb3068), and tectochrysin (pmf0551). The top 10 downregulated metabolites between S_CK and S_T were N-Caffeoyl agmatine (pma0101), L-Alanine (pme1988), kaempferol 3-O-rhamnoside (pme3297), pyridoxal 5'-phosphate (pme1281), sucralose (pmf0574), syringetin (pmb0569), 5-O-hexoside, solanine (pmf0254), diosmin (pmf0549), engeletin (pmf0301), and narirutin (pmf0006) (Figure 4B). 
A

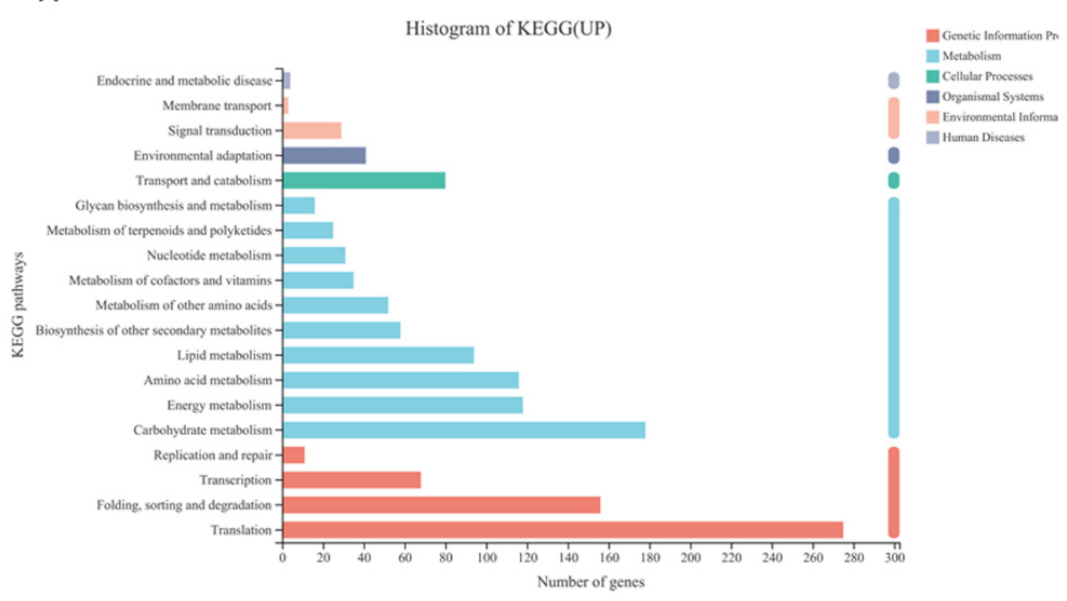

$$
\text { B }
$$

Histogram of KEGG(down)
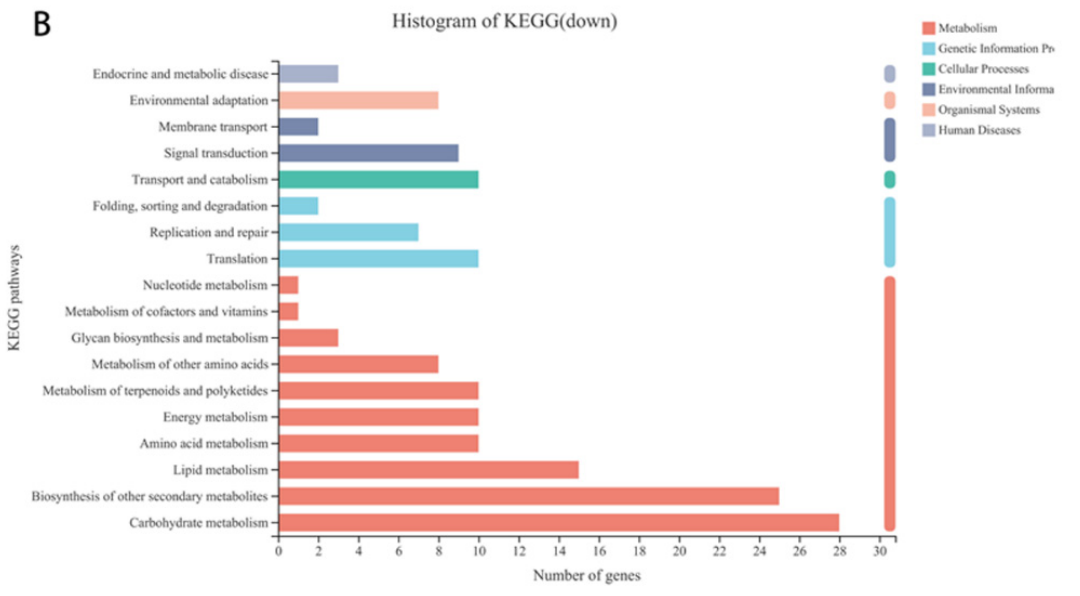

Figure 3. KEGG pathway classification of differentially expressed genes in alfalfa after thrips infection. (A) KEGG pathway classification of upregulated differentially expressed genes in alfalfa after thrips infection. (B) KEGG pathway classification of downregulated differentially expressed genes in alfalfa after thrips infection.
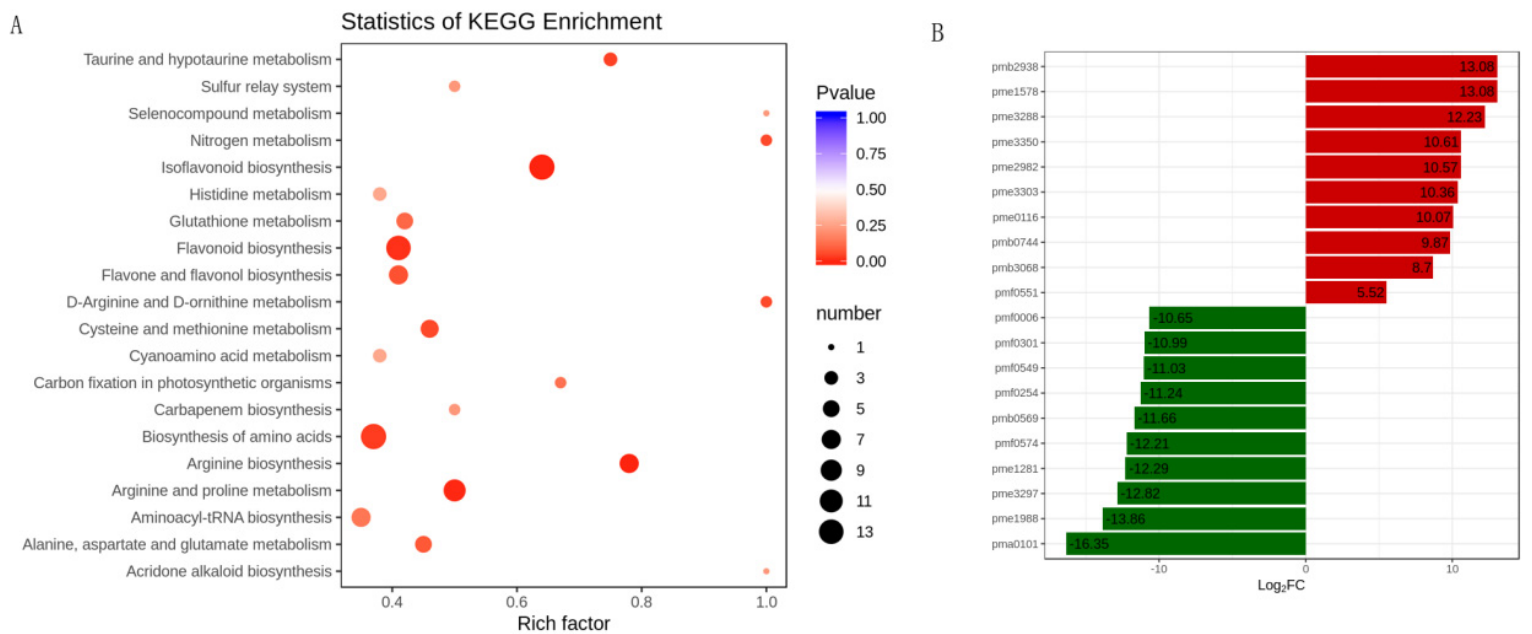

Figure 4. The differentially accumulated metabolites analysis in alfalfa after thrips infection. (A) Scatter plot of KEGG pathways in alfalfa after thrips infection to which the differentially accumulated metabolites were enriched. The degree of enrichment is shown by Rich factor, $p$-value, and the number of metabolites enriched in each pathway; (B) Top 10 up-accumulated (shown in red bars) and top 10 down-accumulated metabolites (shown in green bars) in alfalfa after thrips infection. 


\subsection{Combined Transcriptome and Metabolome Analyses}

To quantitatively map the transcripts directly to metabolic pathways involved in thrips induced resistance, the co-jointKEGG pathway enrichment analysis of transcriptome and metabolome was performed. The results showed that the same pathways of DEGs and DAMs were enriched to flavonoid biosynthesis $(p$-value $<0.05)$ and isoflavonoid biosynthesis ( $p$-value $<0.01$ ) (Figure 5). In order to better understand the relationship between genes and metabolites, the differentially expressed genes and differentially accumulated metabolites were simultaneously mapped to the KEGG pathway diagram (Table S4). As shown in Figure 6A,B, nine key upregulated genes (CYP93C, HI40MT, HIDH, I2' H, IF7MAT, 7-IOMT, VR, CYP81E9, and PTR) and 14 metabolites (11 upregulated and 3 downregulated) were simultaneously mapped to the isoflavonoid biosynthesis (ko00943). In addition, 14 differentially accumulated metabolites and 2 key the differentially expressed genes were simultaneously mapped to the flavonoid biosynthesis (ko00941).

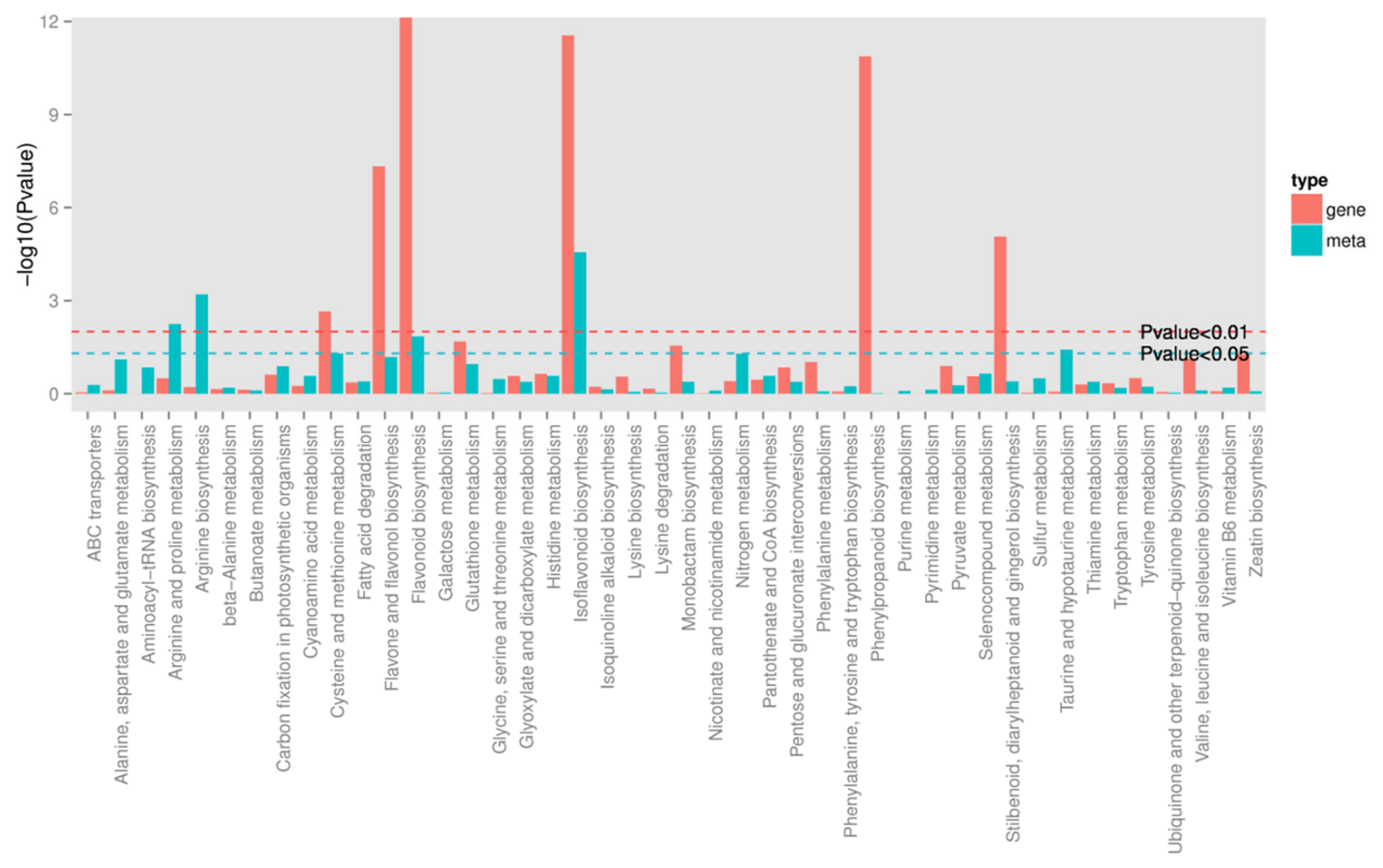

Figure 5. Joint analysis of the differentially expressed genes and differentially accumulated metabolites between S_CK and S_T. Green line represents the selected gene and metabolic pathway at $p$-value $<0.05$, and red line represents the selected gene and metabolic pathway at $p$-value $<0.01$. 

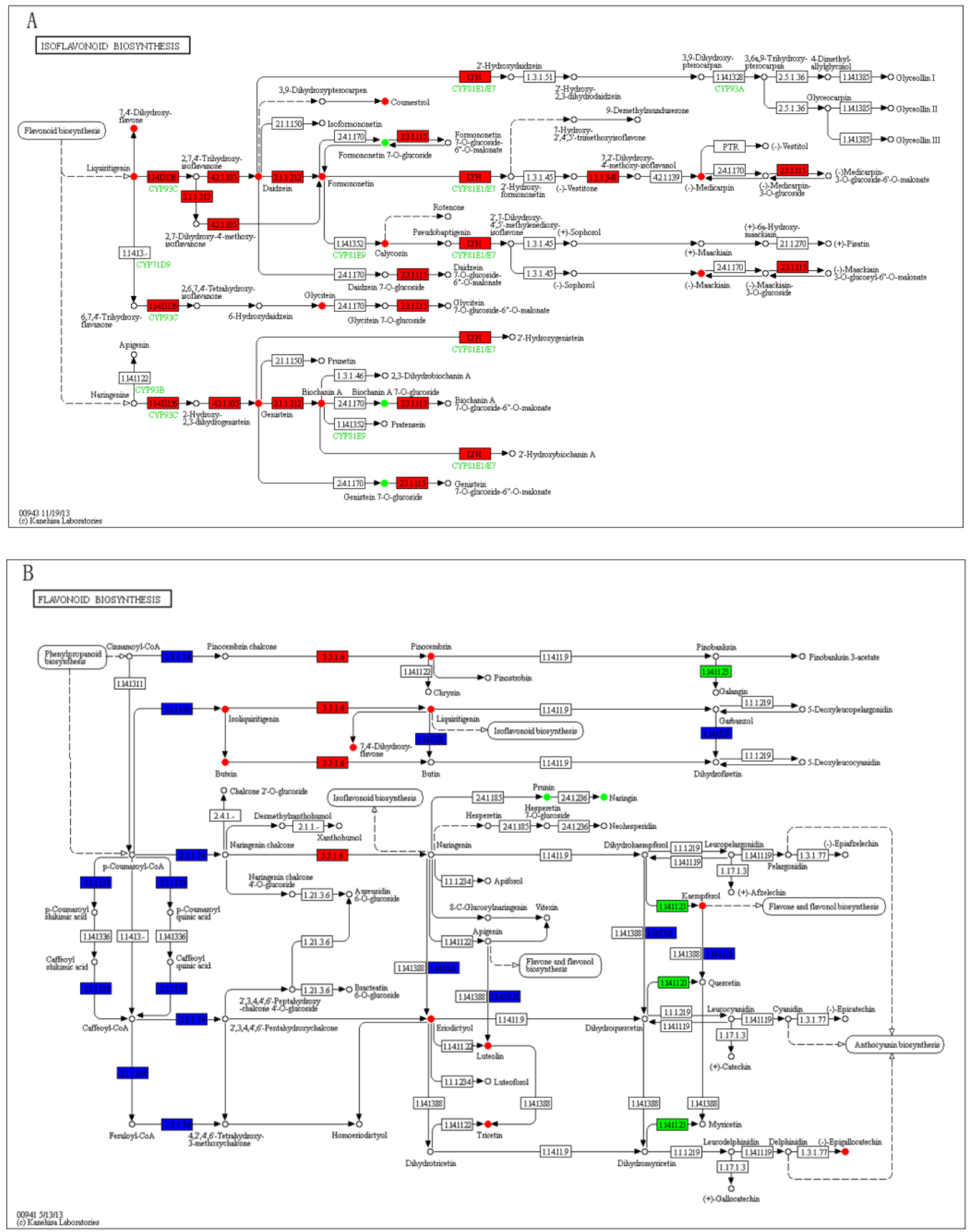

Figure 6. The differentially expressed genes and differentially accumulated metabolites simultaneously mapped to the KEGG pathway diagram. (A) The differentially expressed genes and differentially accumulated metabolites simultaneously mapped to the isoflavonoid biosynthesis (ko00943). (B) The differentially expressed genes and differentially accumulated metabolites simultaneously mapped to the flavonoid biosynthesis (ko00941). Green color represents a significant downregulation of the gene or metabolite, red color represents a significant upregulation of the gene or metabolite, and blue color represents a gene that is both upregulated and downregulated.

\section{Discussion}

Alfalfa is an extremely energy efficient crop and is playing an increasingly important role in low input sustainable agriculture. However, more than 100 insect species damage alfalfa in southeast Asia, northeast Africa, and the U.S. [30]. Thus, it is important to use 
insect-resistant cultivars to control insects that damage both the quantity and quality of the alfalfa. Plants have evolved effective defense mechanisms against insect infestation including morphological traits [31,32], mechanistic (trichomes, hairs) defenses and chemical defenses that involve genes and pathways related to diverse mechanisms [3,33]. Recently, high-quality genome of thrips [34] and various 'omic' technologies [8] have been reported, which may deepen our understanding of the interaction between thrips and plants. For breeding insect resistance and insect control in crops, it is necessary to have information on genetic variation in the host reaction to insect infestation [35]. In this study, we studied the differentially expressed genes and differentially accumulated metabolites based on combined transcriptome and metabolome profiling of S_CK and S_T of Caoyuan No. 2 to explore the mechanisms related to thrips-induced plant resistance.

\subsection{Primary Metabolites Changed Related to Thrips Infestation}

It has been reported that numerous changes in plant primary metabolism, such as carbohydrate and nitrogen metabolism, occur in response to insect attacks [36], and amino acid composition and level affect plant insect resistance [37]. Amino acids are a major source of nitrogen, their content in sap act as a limiting factor in determining the survival of insects [38]. Many plants' defensive compounds are derived from amino acid precursors such as secondary metabolites and glucosinolates [39]. In this study, 23 amino acid and derivatives were significantly changed in alfalfa after thrips infection. Specifically, the upregulated amino acid and derivatives were mainly toxic amino acids or sulphur-amino acid such as L-Carnosine, Phenylacetyl-L-glutamine, L-Cysteine, DL-Homocysteine, $\mathrm{N}$ Phenylacetylglycine, and L-Kynurenine, which might play a crucial role in thrips induced plant defense (Table S3). Consistent with this finding, numerous amino acid metabolism genes related to these amino and derivatives were induced by thrips infestation (Table S2). In addition, plant epicuticular lipid extracts and individual lipid components such as cutin and wax are important for plant insect resistance by affecting oviposition, movement, and feeding [40-42]. One interesting finding in our study was that all the different lipids between S_CK and S_T were downregulated (Table S3). This indicated that lipid levels were negatively correlated with the thrips induced plant defense. Increased photosynthesis and/or local carbohydrate catabolism can serve as energy sources for the production of plant defenses plant-herbivore interactions [43,44]. Consistent with this literature, our study found that many genes involved in energy metabolism (including oxidative phosphorylation and carbon fixation in photosynthetic organisms) and carbohydrate metabolism were induced by thrips infestation (Table S1). These results reveal that primary metabolite (i.e., carbohydrate metabolism, lipid metabolism, and amino acid metabolism) pathways play important roles in thrips induced defense in alfalfa.

\subsection{Hormones Signaling Pathways Related to Thrips Infestation}

Jasmonic acid (JA) and salicylic acid (SA) are the main signal-transduction pathways in plants [45], underlying induced defense against attackers such as herbivorous insects. Both signaling pathways usually act antagonistically, but also have been reported to act synergistically or additively [46,47]. In this study, key genes related to SA transduction including NPR1, TGA, and PR-1 were significantly upregulated (Figure S2). In accordance with the present results, previous studies have demonstrated both thrips resistant and susceptible alfalfa cultivars can regulate gene expression in the SA pathways to enhance plant defense capacity [22]. Thrips feeding activities have been shown to activate the expression of JA-responsive genes [33,48]. Our results showed that thrips feeding activated the JAZ gene (Figure S2), which is one of the key genes in SA biosynthesis. Except for direct damage caused by feeding, thrips also serve as vectors for plant diseases such as tospoviruses. Some findings indicate that viruses can interfere with plant defenses through the interaction of SA with JA signaling [49]. Our results indicated that the SA and JA signaling pathways play important roles in thrips induced plant defense. 


\subsection{Plant Immunity Signaling Pathways Related to Thrips Infestation}

It is well known that plant-pathogen and plant-insects interactions share some responses. Pathogen-associated molecular patterns (PAMPs) and herbivore-associated molecular patterns (HAMPs) are recognized by receptors on the cells in different part of the plant which activate the defense signaling pathways, resulting in a plant's ability to overcome pathogenic invasion and protect against insect predation and damage [47]. We found that the majority of DEGs related to fungal PAPM, bacterial EF-Tu, and bacterial secretion, and that the MAPK signaling pathway-plant were induced in Caoyuan No. 2 after thrips attack (Figures S3 and S4). The activity of these genes would result in plant programmed cell death, the maintenance of the homeostacis, or the accumulation of reactive oxygen species, hypersensitive response, cell wall reinforcement, and stomatal closure and defense-related gene induction. Thus, the thrips induced plant defense occurred. These results indicated that the differentially expressed genes related to MAPK signaling and plant-pathogen interaction may be important for plant-induced defense to thrips, which is consistent with our previous studies and other reports [50,51].

\subsection{Plant Secondary Metabolites Pathway Related to Thrips Infestation}

Plant secondary metabolites, such as alkaloids, glucosinolates, or phenolic compounds, serve as plant defenses against insects [52-54]. However, the content and distribution of individual secondary metabolites vary greatly among plant genotypes [55]. In our study, the results of both transcriptome and metabolome analyses showed that flavonoid biosynthesis and isoflavonoid biosynthesis pathways were induced by thrips injection (Figures 5 and $6 \mathrm{~A}, \mathrm{~B}$ ). This finding is consistent with other studies which observed that both thrips resistant and susceptible alfalfa cultivars can regulate gene expression in the flavonoid biosynthesis pathways to induce defensive genes and protein expression [22] Similar results have been revealed in chickpea and common bean infected with different pathogens [56,57]. Hence, the induction of flavonoid biosynthesis related genes and metabolites in S_T suggested their potential involvement in thrips induced plant defense in alfalfa.

\section{Conclusions}

Thrips feeding could induce several changes in alfalfa. The flavonoid biosynthesis and isoflavonoid biosynthesis pathways were the most significantly enriched pathway in response to thrips infection as shown by the combined transcriptome and metabolome analysis. Plant hormones signal transduction (SA and JA), PAPM-triggered immunity and MAPK signaling pathway-plant pathways played a crucial role in thrips-induced plant resistance in alfalfa. In addition, we found that thrips infestation could also induce numerous changes in plant primary metabolism, such as carbohydrate and amino acid metabolism as compared to the control. Overall, the results described here improve fundamental knowledge of molecular responses to herbivore-inducible plant defense and contribute to the design of strategies against thrips in alfalfa.

Supplementary Materials: The following are available online at https:/ / www.mdpi.com/article/10 .3390/genes12121967/s1, Figure S1: The quadrature signal correction partial least squares-discriminant analysis (OPLS-DA) between S_CK and S_T. Figure S2: Differentially expressed genes between S_CK and S_T related to SA and JA transduction pathway. Figure S3: Differentially expressed genes between S_CK and S_T related to plant-pathogen interaction pathway. Figure S4: Differentially expressed genes between S_CK and S_T related to MAPK signaling pathway-plant pathway. Table S1: All metabolites detected by metabolome. Table S2: Up and downregulated genes between S_CK and S_T annotated to different KEGG pathways. Table S3: Differentially accumulated metabolites between S_CK and S_T. Table S4: The differentially expressed genes and differentially accumulated metabolites and simultaneously mapped to the KEGG pathway diagram (ko00941 and ko00943) in details. 
Author Contributions: Conceptualization, Z.Z. and B.T.; Data curation, Q.C., Y.T. and S.S.; Formal analysis, X.J.; Funding acquisition, Z.Z.; Investigation, Y.T.; Methodology, Y.T., S.S. and R.D.; Resources, X.J. and B.T.; Software, R.D.; Writing—original draft, Z.Z.; and Writing—review and editing, Z.Z. All authors have read and agreed to the published version of the manuscript.

Funding: This work was supported by projects of the National Natural Science Foundation of China (32160333, 31702180), the National Natural Science Foundation of Inner Mongolia, China (2021MS03011) and the Scientific Research Projects in Inner Mongolia, China (NJZY20043).

Institutional Review Board Statement: Not applicable.

Informed Consent Statement: Not applicable.

Data Availability Statement: Data is contained within the article and Supplementary Material.

Acknowledgments: We thank Han Guodong from Inner Mongolia University for helpful comments and suggestions and Andreas Wilkes from England for language editing.

Conflicts of Interest: The authors declare no conflict of interest.

\section{References}

1. Wu, Y.; Temuer, B.; Zhao, X. A study on the alfalfa thrips. Acta Agrestia Sin. 1991, 1, 119-125. (In Chinese)

2. Reitz, S.R. Biology and Ecology of the Western Flower Thrips (Thysanoptera: Thripidae): The Making of a Pest. Fla. Entomol. 2009, 92, 7-13. [CrossRef]

3. Steenbergen, M.; Abd-El-Haliem, A.; Bleeker, P.; Dicke, M.; Escobar-Bravo, R.; Cheng, G.; Haring, M.A.; Kant, M.R.; Kappers, I.; Klinkhamer, P.G.L.; et al. Thrips advisor: Exploiting thrips-induced defences to combat pests on crops. J. Exp. Bot. 2018, 69, 1837-1848. [CrossRef] [PubMed]

4. Yang, Q. Guide of Alfalfa Production and Management; Forestry: Beijing, China, 2003.

5. Reitz, S.R.; Gao, Y.; Kirk, W.D.J.; Hoddle, M.S.; Leiss, K.A.; Funderburk, J.E. Invasion Biology, Ecology and Management of Western Flower Thrips. Annu. Rev. Entomol. 2020, 51, 17-37. [CrossRef] [PubMed]

6. Howe, G.A.; Jander, G. Plant immunity to insect herbivores. Annu. Rev. Plant Biol. 2008, 59, 41-66. [CrossRef]

7. War, A.R.; Paulraj, M.G.; Ahmad, T.; Buhroo, A.A.; Hussain, B.; Ignacimuthu, S.; Sharma, H.C. Mechanisms of plant defense against insect herbivores. Plant Signal. Behav. 2012, 7, 1306-1320. [CrossRef]

8. Züst, T.; Agrawal, A.A. Trade-Offs between Plant Growth and Defense against Insect Herbivory: An Emerging Mechanistic Synthesis. Annu. Rev. Plant Biol. 2017, 68, 513-534. [CrossRef] [PubMed]

9. Chen, T.Y.; Chu, C.C.; Henneberry, T.J. Frankliniella occidentalis colonization on okra- and normal-leaf cotton strains and cultivars. Southwest. Entomol. 2006, 31, 281-287.

10. Khan, M.A.; Ali, A.; Aslam, M.; Tahir, Z.; Khan, M.M.; Nadeem, I. The role of morphological and chemical plant traits imparting resistance in bt cotton genotypes against thrips, Thrips tabaci (Lind.). Pak. J. Agric.-Cult. Sci. 2014, 51, 725-731.

11. Miyazaki, J.; Stiller, W.N.; Wilson, L.J. Sources of plant resistance to thrips: A potential core component in cotton IPM. Entomol. Exp. Appl. 2017, 162, 30-40. [CrossRef]

12. Kariyat, R.R.; Balogh, C.M.; Moraski, R.P.; De Moraes, C.M.; Mescher, M.C.; Stephenson, A.G. Constitutive and herbivore-induced structural defenses are compromised by inbreeding in Solanum carolinense (Solanaceae). Am. J. Bot. 2013, 100, 1014-1021. [CrossRef] [PubMed]

13. Khorramdelazad, M.; Bar, I.; Whatmore, P.; Smetham, G.; Bhaaskaria, V.; Yang, Y.; Bai, S.H.; Mantri, N. Transcriptome profiling of lentil (Lens culinaris) through the first 24 hours of Ascochyta lentis infection reveals key defence response genes. BMC Genom. 2018, 19, 108. [CrossRef] [PubMed]

14. Ahmed, A.M.M.; Solorio Sanchez, F.J.; Ramirez, Y.; Aviles, L.; Mahdy, R.E.E.; Castillo Camaal, J.B. Tannins and mi-mosine in Leucaena genotypes and their relations to Leucaena resistance against Leucaena Psyllid and Onion thrips. Agrofor. Syst. 2017, 91, 1-8. [CrossRef]

15. Hurej, M.; Kucharczyk, H.; Twardowski, J.P.; Kotecki, A. Thrips (Thysanoptera) associated with two genetically modified types of linseed (Linum usitatissimum L.). J. Plant Dis. Prot. 2017, 124, 81-91. [CrossRef]

16. Bertea, C.M.; Casacci, L.P.; Bonelli, S.; Zampollo, A.; Barbero, F. Chemical, Physiological and Molecular Responses of Host Plants to Lepidopteran Egg-Laying. Front. Plant Sci. 2020, 10, 1768. [CrossRef]

17. Mellway, R.D.; Constabel, C.P. Metabolic engineering and potential functions of proanthocyanidins in poplar. Plant Signal. Behav. 2009, 4, 790-802. [CrossRef] [PubMed]

18. Mirnezhad, M.; Romero-González, R.R.; Leiss, K.A.; Choi, Y.H.; Verpoorte, R.; Klinkhamer, P.G.L. Metabolomic Analysis of Host Plant Resistance to Thrips in Wild and Cultivated Tomatoes. Phytochem. Anal. 2010, 21, 110-117. [CrossRef] [PubMed]

19. Yang, T.; Stoopen, G.; Wiegers, G.; Mao, J.; Wang, C.; Dicke, M.; Jongsma, M.A. Pyrethrins Protect Pyrethrum Leaves Against Attack by Western Flower Thrips, Frankliniella occidentalis. J. Chem. Ecol. 2012, 38, 370-377. [CrossRef]

20. Wang, X.-S.; Yang, C.-L.; Wang, S.-S.; Hu, G.-X. Changes of phenols and lignin contents in alfalfa leaf damaged by Odontothrips loti. J. Appl. Ecol. 2014, 25, 1688-1692. (In Chinese) 
21. Liu, X.; Klinkhamer, P.G.; Vrieling, K. The effect of structurally related metabolites on insect herbivores: A case study on pyrrolizidine alkaloids and western flower thrips. Phytochemistry 2017, 138, 93-103. [CrossRef]

22. Tu, X.; Liu, Z.; Zhang, Z. Comparative transcriptomic analysis of resistant and susceptible alfalfa cultivars (Medicago sativa L.) after thrips infestation. BMC Genom. 2018, 19, 116. [CrossRef]

23. Fracasso, A.; Trindade, L.M.; Amaducci, S. Drought stress tolerance strategies revealed by RNA-Seq in two sorghum genotypes with contrasting WUE. BMC Plant Biol. 2016, 16, 115. [CrossRef]

24. Wang, Y.; Zeng, X.; Liu, W. De novo transcriptomic analysis during Lentinula edodes fruiting body growth. Gene 2018, 641, 326-334. [CrossRef] [PubMed]

25. Lin, Y.; Wang, Y.; Li, B.; Tan, H.; Li, D.; Li, L.; Liu, X.; Han, J.; Meng, X. Comparative transcriptome analysis of genes involved in anthocyanin synthesis in blueberry. Plant Physiol. Bioch. 2018, 127, 561-572. [CrossRef] [PubMed]

26. Cock, P.J.A.; Fields, C.J.; Goto, N.; Heuer, M.L.; Rice, P.M. The Sanger FASTQ file format for sequences with quality scores, and the Solexa/Illumina FASTQ variants. Nucleic Acids Res. 2010, 38, 1767-1771. [CrossRef] [PubMed]

27. Chen, W.; Gong, L.; Guo, Z.; Wang, W.; Zhang, H.; Liu, X.; Yu, S.; Xiong, L.; Luo, J. A Novel Integrated Method for Large-Scale Detection, Identification, and Quantification of Widely Targeted Metabolites: Application in the Study of Rice Metabolomics. Mol. Plant 2013, 6, 1769-1780. [CrossRef] [PubMed]

28. Kanehisa, M.; Goto, S. KEGG: Kyoto encyclopedia of genes and genomes. Nucleic Acids Res. 2000, 28, 27-30. [CrossRef] [PubMed]

29. Jozefczuk, S.; Klie, S.; Catchpole, G.; Szymanski, J.; Cuadros-Inostroza, A.; Steinhauser, D.; Selbig, J.; Willmitzer, L. Metabolomic and transcriptomic stress response of Escherichia coli. Mol. Syst. Biol. 2010, 6, 364. [CrossRef] [PubMed]

30. Clement, S.L.; Quisenberry, S.S. Global Plant Genetic Resources for Insect-Resistant Crops; CRC Press: Boca Raton, NY, USA, 1999.

31. Kaur, B.; Kuraparthy, V.; Bacheler, J.; Fang, H.; Bowman, D.T. Screening Germplasm and Quantification of Com-ponents Contributing to Thrips Resistance in Cotton. J. Econ. Entomol. 2018, 111, 2426-2434. [CrossRef] [PubMed]

32. Jacob, T.K.; Senthil Kumar, C.M.; Devasahayam, S.; D’Silva, S.; Kumar, R.S.; Biju, C.N.; Praveena, R.; Ankegowda, S.K.J. Plant morphological traits associated with field resistance to cardamom thrips (Sciothrips cardamomi) in cardamom (Elettaria cardamomum). Ann. Appl. Biol. 2020, 177, 143-151. [CrossRef]

33. Escobar-Bravo, R.; Klinkhamer, P.G.L.; Leiss, K.A. Induction of Jasmonic Acid-Associated Defenses by Thrips Alters Host Suitability for Conspecifics and Correlates with Increased Trichome Densities in Tomato. Plant Cell Physiol. 2017, 58, 622-634. [CrossRef] [PubMed]

34. Guo, S.-K.; Cao, L.-J.; Song, W.; Shi, P.; Gao, Y.-F.; Gong, Y.-J.; Chen, J.-C.; Hoffmann, A.A. Chromosome-level assembly of the melon thrips genome yields insights into evolution of a sap-sucking lifestyle and pesticide resistance. Mol. Ecol. Resour. 2020, 20, 1110-1125. [CrossRef] [PubMed]

35. Srinivasan, R.; Abney, M.R.; Lai, P.-C.; Culbreath, A.K.; Tallury, S.; Leal-Bertioli, S.C.M. Resistance to Thrips in Peanut and Implications for Management of Thrips and Thrips-Transmitted Orthotospoviruses in Peanut. Front. Plant Sci. 2018, 9, 1604. [CrossRef]

36. Zhou, S.; Lou, Y.-R.; Tzin, V.; Jander, G. Alteration of Plant Primary Metabolism in Response to Insect Herbivory. Plant Physiol. 2015, 169, 1488-1498. [CrossRef] [PubMed]

37. Abidallha, E.H.M.A.; Li, Y.; Li, H.; Chen, Y.; Tambel, L.I.M.; Hu, D.; Zhang, X.; Chen, D. Amino acid composition and level affect Bt protein concentration in Bt cotton. Plant Growth Regul. 2017, 82, 439-446. [CrossRef]

38. Tsumuki, H.K.K.; Shiraga, T.; Kawada, K. Characteristics of barley resistance to cereal aphids. Nutritional differences between barley strains. Nogaku Kenkyu 1987, 61, 149-159. [CrossRef]

39. Fan, J.; Crooks, C.; Creissen, G.; Hill, L.; Fairhurst, S.; Doerner, P.; Lamb, C. Pseudomonas sax Genes Overcome Aliphatic Isothiocyanate-Mediated Non-Host Resistance in Arabidopsis. Science 2011, 331, 1185-1188. [CrossRef] [PubMed]

40. Karmakar, A.; Malik, U.; Barik, A. Effects of leaf epicuticular wax compounds from Solena amplexicaulis (Lam.) Gandhi on olfactory responses of a generalist insect herbivore. Allelopath. J. 2016, 37, 253-272.

41. Reina-Pinto, J.J.; Yephremov, A. Surface lipids and plant defenses. Plant Physiol. Biochem. 2009, 47, 540-549. [CrossRef] [PubMed]

42. Zogli, P.; Pingault, L.; Grover, S.; Louis, J. Ento(o)mics: The intersection of "omic" approaches to decipher plant defense against sap-sucking insect pests. Curr. Opin. Plant Biol. 2020, 56, 153-161. [CrossRef] [PubMed]

43. Botha, A.M.; Lacock, L.; Van Niekerk, C.; Matsioloko, M.T.; Du Preez, F.B.; Loots, S.; Venter, E.; Kunert, K.J. Is photosynthetic transcriptional regulation in Triticum aestivum L. cv. "TugelaDN" a contributing factor for tolerance to Diuraphis noxia (Homoptera: Aphididae)? Plant Cell Rep. 2006, 25, 41-54. [CrossRef] [PubMed]

44. Gutsche, A.; Heng-Moss, T.; Sarath, G.; Twigg, P.; Xia, Y.; Lu, G.; Mornhinweg, D. Gene expression profiling of tolerant barley in response to Diuraphis noxia (Hemiptera: Aphididae) feeding. Bull. Entomol. Res. 2009, 99, 163-173. [CrossRef]

45. Sarde, S.J.; Bouwmeester, K.; Venegas-Molina, J.; David, A.; Boland, W.; Dicke, M. Involvement of sweet pepper CaLOX2 in jasmonate-dependent induced defence against Western flower thrips. J. Integr. Plant Biol. 2019, 61, 1085-1098. [CrossRef]

46. Pieterse, C.M.J.; Van Der Does, D.; Zamioudis, C.; Leon-Reyes, A.; Van Wees, S.C.M. Hormonal Modulation of Plant Immunity. Annu. Rev. Cell Dev. Biol. 2012, 28, 489-521. [CrossRef]

47. Thaler, J.S.; Humphrey, P.T.; Whiteman, N.K. Evolution of jasmonate and salicylate signal crosstalk. Trends Plant Sci. 2012, 17, 260-270. [CrossRef] [PubMed]

48. Selig, P.; Keough, S.; Nalam, V.J.; Nachappa, P. Jasmonate-dependent plant defenses mediate soybean thrips and soybean aphid performance on soybean. Arthropod-Plant Interact. 2016, 10, 273-282. [CrossRef] 
49. Shalileh, S.; Ogada, P.A.; Moualeu, D.P.; Poehling, H.-M. Manipulation of Frankliniella occidentalis (Thysanoptera: Thripidae) by Tomato Spotted Wilt Virus (Tospovirus) Via the Host Plant Nutrients to Enhance Its Transmission and Spread. Environ. Entomol. 2016, 45, 1235-1242. [CrossRef] [PubMed]

50. Temuer, B.; Si, Q. Anti-thrips alfalfa form features andf anti-insect sex research. J. Inn. Mong. Agric. Univ. 2014, 35, 51-58. (In Chinese)

51. Malik, N.a.A.; Kumar, I.S.; Nadarajah, K. Elicitor and Receptor Molecules: Orchestrators of Plant Defense and Immunity. Int. J. Mol. Sci. 2020, 21, 963. [CrossRef] [PubMed]

52. Lopez-Goldar, X.; Villari, C.; Bonello, P.; Borg-Karlson, A.K.; Grivet, D.; Zas, R.; Sampedro, L. Inducibility of Plant Secondary Metabolites in the Stem Predicts Genetic Variation in Resistance Against a Key Insect Herbivore in Maritime Pine. Front. Plant Sci. 2018, 9, 1651. [CrossRef] [PubMed]

53. Ren, X.; Wu, S.; Xing, Z.; Gao, Y.; Cai, W.; Lei, Z. Abundances of thrips on plants in vegetative and flowering stages are related to plant volatiles. J. Appl. Entomol. 2020, 144, 732-742. [CrossRef]

54. Wink, M. Plant Secondary Metabolites Modulate Insect Behavior-Steps Toward Addiction? Front. Physiol. 2018, 9, 364. [CrossRef]

55. Soltis, N.E.; Kliebenstein, D.J. Natural Variation of Plant Metabolism: Genetic Mechanisms, Interpretive Caveats, and Evolutionary and Mechanistic Insights. Plant Physiol. 2015, 169, 1456-1468. [CrossRef]

56. Kumar, Y.; Zhang, L.; Panigrahi, P.; Dholakia, B.B.; Dewangan, V.; Chavan, S.G.; Kunjir, S.M.; Wu, X. Fusarium oxysporum mediates systems metabolic reprogramming of chickpea roots as revealed by a combination of proteomics and metabolomics. Plant Biotechnol. J. 2016, 14, 1589-1603. [CrossRef]

57. Chen, L.; Wu, Q.; He, W.; He, T.; Wu, Q.; Miao, Y. Combined De Novo Transcriptome and Metabolome Analysis of Common Bean Response to Fusarium oxysporum f. sp. phaseoli Infection. Int. J. Mol. Sci. 2019, 20, 6278. [CrossRef] [PubMed] 